\title{
Optimal Advertising Budget Allocation across Markets with Different Goals and Various Constraints
}

\author{
Xingyuan Wang $\left(\mathbb{D},{ }^{1}\right.$ Fuan $\mathrm{Li}^{2}$ and Fan Jia $\mathbb{D}^{3}$ \\ ${ }^{1}$ School of Management, Shandong University, Jinan, Shandong 250100, China \\ ${ }^{2}$ Department of Marketing \& Management Sciences, William Paterson University, 1600 Valley Road, Wayne, NJ 07470, USA \\ ${ }^{3}$ School of Management Science and Engineering, Shandong University of Finance and Economics, Jinan, \\ Shandong 250014, China \\ Correspondence should be addressed to Fan Jia; fanta07@126.com
}

Received 2 July 2019; Revised 10 April 2020; Accepted 15 April 2020; Published 21 May 2020

Academic Editor: Hassan Zargarzadeh

Copyright ( 92020 Xingyuan Wang et al. This is an open access article distributed under the Creative Commons Attribution License, which permits unrestricted use, distribution, and reproduction in any medium, provided the original work is properly cited.

\begin{abstract}
Advertising budget allocation across multiple markets has drawn considerable attention in recent years. To expand previous research and fill a gap in the current literature, this study proposes two decision models for optimal budget allocation decisions across multimarkets with different goals and various constraints. In addition to the market parameters proposed by the Vidale-Wolfe model, the present study incorporates market goals and advertising objectives into budget allocation decisions. Different types of markets are defined in terms of the goal set for market share or profit. Given the characteristics of different markets, two separate decision models are developed. Model I aims to maximize sales volume given a fixed advertising budget, while model II seeks to minimize the advertising budget given a total of targeted sales volume for all the markets. Solutions to the two models are discussed, and a numerical example is provided to demonstrate how to apply the models in making budget allocation decision.
\end{abstract}

\section{Introduction}

Advertising expenditure has long been an important concern for both marketing practitioners and academicians because of its direct impact on sales and profit. Among the earliest and most influential academic studies are the work by Vidale and Wolfe [1] who developed a sales response model to advertising and Dorfman and Steiner [2] who described the necessary condition for budgeting problems aiming at maximizing corporate profit. Previous research falls roughly into two major categories-advertising response models and advertising decision models. Well known in the first category are models developed by Vidal-Wolfe [1], Dorfman and Steiner [2], Nerlove and Arrow [3], Sethi [4, 5], Clarke [6], and Freidman [7], among others. Unlike advertising response models that focus on tracking and assessing advertising effectiveness and diagnosing market changes, models in the second category devote to advertising decisions, especially those concerning annual budget, geographic distribution, timing, and the like [8-11]. Little [12] pointed out that understanding consumers' response to advertising is essential to advertising decisions. Thus, scholars frequently built budget decision models based on advertising response models $[7,9,13]$. No doubt, all these models have greatly contributed to our understanding of advertising response and budget allocation decisions. Nevertheless, the focus of those studies remains on single products and/or a single market [14]. Actually, advertising models are dynamic, which reflected in both dynamic markets and dynamic advertising policy [15].

For advertising models with dynamic markets, it was not until recently that researchers started to examine budget decisions for multiproduct and multimarket allocation [14, 16, 17]. Tull et al. [18] indicated that profit improvement from better allocation across products or regions is much higher than from simply increasing the overall budget. Taking an integrated allocation approach, Fisher et al. [14] proposed and empirically tested budget allocation models across products and countries. The decision model allows determining nearly optimal marketing budgets at the country-product-marketing activity levels. Even though the proposed model does not solely focus on advertising budget, this study is important because it considers a 
budget decision involving multiproducts and multimarkets. Besides Fisher et al. [14], recent studies have examined resource allocation across customers $[19,20]$ or sales territories [21]. Abedi [22] considered multiple channels and multiple markets and formulated a nonlinear and nonseparable knapsack problem to obtain the solution of advertising budget allocation. Muneeb et al. [23] presented bilevel decision-making models so as to allocate advertisements to different channels under different time zones of a day with the objectives of maximization of ads' impact and minimization of net cost at two different levels. Eshghi et al. [24] established a diffusion dynamic model to analyze optimal strategies for the allocation of a finite budget that can be invested in different advertising channels. In addition, Aravindakshan et al. [25] studied budget allocation between national and regional advertising, and Dube and Manchanda [26] examined budget allocation across regional markets in response to competition.

For advertising models with dynamic policy, advertising models have been established by the optimal control method from different viewpoints. Liu et al. [27] investigated effects of negotiated and administered transfer pricing on the profits of each center and firm and proposed the strategic transfer pricing in a marketing-operation interface. Feng et al. [28] proposed a dynamic pricing model for perishable produces and obtained the analytical solutions of the joint dynamic pricing and advertising policy. Lu et al. [29] considered the reference price effect on joint pricing and advertising strategy in the monopolistic firm and established an optimization model to obtain maximal profits. Aliaga et al. [30] extended Vidale-Wolfe and Lanchester models of duopoly dynamics by introducing a third population of undecided users and established equilibrium condition and the stability properties under different classes of advertising policies. With the rapid development of global market economy, companies inevitably face multiple heterogeneous markets, and decision makers have to consider different characteristics of markets in order to obtain reasonable allocation of advertising budget. Wu et al. [31] studied the coordinating pricing and advertising decisions for the supply chain under the consignment contract and proposed the optimal pricing and advertising strategies in the decentralized and centralized scenarios by using differential game theory. $\mathrm{Lu}$ et al. [32] considered a bilateral monopoly supply chain and compared partially myopic and forward-looking strategies in the decentralized scenario and centralized scenario and then developed a differential game in a two-player supply chain with sticky price where a manufacturer provided a cooperative advertising program for a retailer [33]; by considering the effect of dynamic advertising, Lu et al. [34] investigated two distinct types of contracts between a dominant retailer and a manufacturer. Nie and Zhang [35] studied the effect of advertising productiveness difference on the distribution channel selection by a manufacturer and obtained the results that, with high initial goodwill or advertising productiveness, the manufacturer should develop its own channel.

Although research on budget allocation across markets and product categories is still limited, these studies represent significant progress in such research. The present study is an attempt to expand this effort and fill a research gap in the current literature. Because of the advance of transportation, network technology, and the globalization of the world economy, businesses today are often marketing in multiple heterogeneous markets. It is especially true of transnational companies. As a result, managers must consider different characteristics of those markets when making their budget decisions. They must meet the challenge of effectively and efficiently coordinating and integrating advertising efforts while breaking a global advertising budget into individual budget for different markets. In other words, marketers must coordinate all advertising efforts to achieve optimal use of limited resources while considering the heterogeneous conditions across multiple markets. When a company has business in multicountries or regions, its goals and strategies may vary significantly depending on the market characteristics and the nature of their presence in the particular market, whether a country or a region. Inevitably, managers must incorporate their strategic considerations into advertising budget decisions. For instance, for a new market where a company has no presence or only a weak presence, market development is its primary concern, and consequently, the focus is on building sales volume and gaining market share. In contrast, for a mature market where it has already a strong presence, the focus may shift to how to maximize profit while maintaining current market share. Unfortunately, such strategic consideration has received little attention in the extant budget allocation literature. To our knowledge, there is no method solving the multiconstraint budget-allocation problem up to date. In reality, markets of unique conditions place specific constraints on advertising campaigns. Consequently, managers need to find a feasible method to solve the multiconstraint advertising budget problem. Attempting to fill the literature gap, we have developed a model based on the Vidale-Wolfe advertising response model, which explicitly considers the relationship between market conditions, saturation level, market responses, and advertising spending. We incorporate market conditions and specific objectives (strategic concerns) for the markets into budget allocation consideration and develop two separate decision models for advertising budget allocation under different constraints. In short, the decision problem we attempt to address is how to allocate advertising resources under different constraints for multiple markets with varying market goals and objectives.

The rest of the paper begins with a brief introduction to the Vidale-Wolfe model and then discusses two different types of markets. Next, decision models for budget allocation across multimarkets under different constraints are presented. After discussing the characteristics of the two decision models, it turns to the solutions to the models and a numerical example that illustrates the application of such models. The paper concludes with brief discussion of implications and future research direction.

\section{Model Formulations}

The notations given in Table 1 are used in the entire paper.

2.1. Vidale-Wolfe Models. As one of the earliest studies on advertising responses, Vidale and Wolfe developed a simple 
TABLE 1: Explanations of notations used in this paper.

\begin{tabular}{lc}
\hline Notation & Explanation \\
\hline$t$ & Time \\
$s(t)$ & The rate of sales at time $t$ \\
$a(t)$ & The rate of advertising expenditure \\
$\lambda$ & Sales decay constant \\
$M$ & Saturation level \\
$r$ & Response constant \\
$c$ & Advertising expenditure \\
$S$ & The total sales in the period $\left[t_{0}, T\right]$ \\
$z$ & The expected level of market share \\
$p$ & Net profit per unit before the advertising campaign \\
$A$ & The set of all the new markets \\
\hline
\end{tabular}

model about the interaction of advertising and sales, a model that is consistent with their experimental data concerning a large number of products and several media. Vidale and Wolfe argued that the change in rate of sales depends on three parameters: sales decay constant $\lambda$, saturation level $M$, and response constant $r$ [3]. The Vidal-Wolfe model is represented by

$$
\frac{\mathrm{d} s(t)}{\mathrm{d} t}=r a(t) \frac{M-s(t)}{M}-\lambda s(t),
$$

where $s(t)$ is the rate of sales at time $t$ and $a(t)$ is the rate of advertising expenditure.

In the case of a fixed budget and a fixed advertising duration, differences in the advertising mode as well as timing can have drastic influences on the effectiveness of the advertising campaign. Given the complexity involved and for the sake of lack of existing models, we follow the previous study in [36], assuming that advertising budget is evenly distributed through the duration of the campaign. Thus, the rate of advertising expenditure $c$ can be simplified as a constant, that is,

$$
a(t)= \begin{cases}\frac{c}{\theta}, & t_{0} \leq t \leq t_{0}+\theta \\ 0, & t_{0}+\theta<t\end{cases}
$$

where $\theta$ is the duration of the advertising campaign. According to equation (2), the Vidale-Wolfe model can be simplified as follows:

$$
\begin{aligned}
& \frac{\mathrm{d} s(t)}{\mathrm{d} t}=\frac{r c}{\theta}-\frac{r c+\lambda M \theta}{M \theta} s(t), \quad t_{0} \leq t \leq t_{0}+\theta, \\
& \frac{\mathrm{d} s(t)}{\mathrm{d} t}=-\lambda s(t), \quad t_{0}+\theta<t .
\end{aligned}
$$

Equation (3) depicts the interaction of sales and advertising during the campaign, while equation (4) describes the rate of sales after the advertising campaign comes to an end.

Let $s\left(t_{0}\right)=\mu$ at $t=0$, the time when the advertising campaign starts; we obtain the following by finding the differential equation for equation (3):

$$
s_{1}(t)=\frac{M r c}{r c+\lambda M \theta}+e^{-(r c+\lambda M \theta / M \theta)\left(t-t_{0}\right)} e^{-(r c+\lambda M \theta / M \theta)\left(t-t_{0}\right)}, \quad t_{0} \leq t \leq t_{0}+\theta .
$$

Then, the rate of sales at the end of the advertising campaign is

$$
s\left(t_{0}+\theta\right)=\frac{M r c}{r c+\lambda M \theta}+\left(\mu-\frac{M r c}{r c+\lambda M \theta}\right) e^{-(r c+\lambda M \theta / M)} .
$$

Taking equation (6) as the initial condition of equation (4), we can get

$$
s_{2}(t)=\left(\frac{M r c}{r c+\lambda M \theta}\left(e^{\lambda \theta}-e^{-(r c / M)}\right)+\mu e^{-(r c / M)}\right) e^{\lambda t_{0}} e^{-\lambda t}, \quad t_{0}+\theta<t .
$$

Let $S$ be the total sales in the period $\left[t_{0}, T\right]$; then, total sales $S$ can be calculated and expressed as

$$
\begin{aligned}
S= & \int_{t_{0}}^{T} s(t) \mathrm{d} t=\int_{t_{0}}^{t_{0}+\theta} s_{1}(t) \mathrm{d} t+\int_{t_{0}+\theta}^{T} s_{2}(t) \mathrm{d} t \\
= & \int_{t_{0}}^{t_{0}+\theta} \frac{M r c}{r c+\lambda M \theta}+\left(\mu-\frac{M r c}{r c+\lambda M \theta}\right) e^{-(r c+\lambda M \theta / M \theta)\left(t-t_{0}\right)} \mathrm{d} t+\int_{t_{0}+\theta}^{T}\left(\frac{M r c}{r c+\lambda M \theta}\left(e^{\lambda \theta}-e^{-(r c / M)}\right)+\mu e^{-(r c / M)}\right) e^{\lambda t_{0}} e^{-\lambda t} \mathrm{~d} t \\
= & \frac{\theta M\left(\mu r c-r c M+\mu \lambda M \theta+r^{2} c^{2}+r c \lambda \theta M-e^{-(r c+\lambda M \theta / M)}(\theta \mu \lambda M+\mu r c-r c M)\right)}{(r c+\lambda M \theta)^{2}} \\
& +\left(\frac{M r c}{r c+\lambda M \theta}\left(e^{\lambda \theta}-e^{-(r c / M)}\right)+\mu e^{-(r c / M)}\right) \frac{e^{-\lambda \theta}-e^{-\lambda\left(T-t_{o}\right)}}{\lambda} .
\end{aligned}
$$


2.2. Two Types of Markets with Different Market Goals. Businesses often sell their products or services in multiple heterogeneous markets. Differences among those markets make it necessary that managers consider the actual conditions of various markets while making advertising budget decisions. Differences in market conditions lie in either characteristics of markets defined by sales decay constant $\lambda$, saturation level $M$, and response constant $r$ or market position (sales status) of the firm defined by sales rate, sales volume, and market share. The general principle or objective of advertising decisions is to achieve maximum gain with minimal expenditure. However, when it comes to multiple markets where a firm's market positions differ, managers may want to set specific goals and objectives for each market according to specific market characteristics and its market positions.

There are generally two special types of markets: first, type A market, a new market to the firm where it has no presence (no sales or market share) or has only weak presence (i.e., low sales volume and small market share) and second, type B market, a mature market to the firm where it has a strong presence, namely, it has reached the desired level of sales volume and market share. For the first type of market, the primary concern (or strategic goal) of the company is to develop the market and/ or strengthen the market position. Thus, the objective of advertising is to increase sales and maximize market share. In contrast, in a mature market in which it has achieved its sales goals and desirable level of market share, its primary concern (goal) may have shifted to profitability. The objective of advertising becomes to maintain market share and maximize profit, while controlling advertising expenditure. Therefore, for companies that market in both types of markets, managers must make budget decision in terms of their market goals, advertising objectives, and corresponding specific constraints for each market while trying to maximize gain at minimum cost (see Figure 1). Accordingly, optimal budget allocation should be defined in terms of the extent to which it promotes or contributes to the market goals and objectives.

2.2.1. Type A: New Market with the Market Share Goal. For type A market in which market development is the primary goal, managers often rely on extensive advertising campaigns to create brand awareness, change consumer attitude and preference, induce product sales, and increase market share. Thus, the objectives of the advertising budget decision for type A markets are to build market share to a level that meets or exceeds an expected value $z$ at the measuring time $T$. The formula can be expressed as

$$
\frac{s(T)}{M} \geq z
$$

where $s(T)$ is the rate of sales at time $T, M$ is the saturation level in the Vidale-Wolfe model, and $z$ is the expected level of market share where $0<z \leq 1$.

For an advertising campaign involving multiple type A markets, we let $A$ indicate the set of all the new markets; then, for every market $i \in A$, there is $\left(s_{i}(T) / M_{i}\right) \geq z_{i}$, where $s_{i}(T), M_{i}$, and $z_{i}$ are the rate of sales at time $T$, saturation level, and expected share of market $i$, respectively.

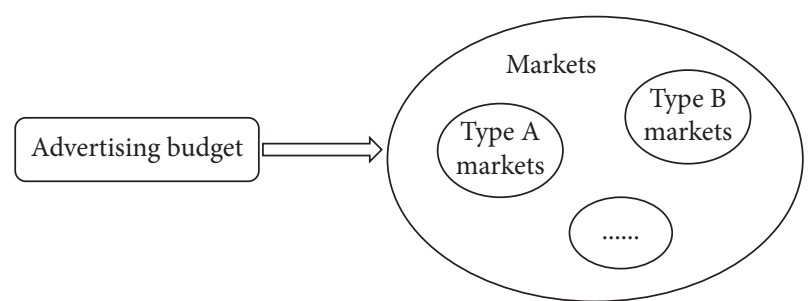

FIgUre 1: Two special types of markets in advertising budget allocation.

\subsubsection{Type B: Mature Market with the Market Profit Goal.} For markets in which the company has achieved desired level of brand awareness, sales volume, and has built loyal customer base, building market share is no longer the primary goal for the company. In fact, as the marginal utility of advertising in a type $\mathrm{B}$ market becomes lower than it is in a type A market, it is more desirable that the company shifts its focus to profitability. The objective of budget allocation then becomes to achieve or exceed an expected value of profit in type B markets. Symbolically,

$$
p S-c \geq l,
$$

where $p$ is the net profit per unit before the advertising campaign, $S$ is the total sales volume between starting time $t_{0}$ and measuring time $T, c$ is the total cost of the advertising campaign, and $l$ is the expected profit.

Let $B$ represent the set of type B markets in question; then, there is $p_{j} S_{j}-c_{j} \geq l_{j}$ for every $j \in B$, where $p_{j}, S_{j}, c_{j}$, and $l_{j}$ are the net profit per unit, the total sales volume between starting time $t_{0}$ and measuring time $T$, the total cost of the advertising campaign, and expected profit of market $j$, respectively.

2.3. Budget Allocation Models for Multimarkets under Different Constraints. When allocating advertising budget for multimarkets of heterogeneous conditions, business managers must consider not only the market characteristics but also its sales status and specific market goals and advertising objectives. Differences in market responses to adverting affected by sale decay constant $\lambda$, saturation level $M$, and response constant $r$, along with differences in specific market goals and adverting objectives, impose specific constraints on the decision makers. In view of different constraints of the two types of markets, we propose two separate decision models for budget allocation across multimarkets with specific advertising objectives.

Suppose that the total budget $C$ is allocated among $k$ target markets of an advertising campaign, where we label the markets as $1,2, \ldots, k$. Let $\theta$ be the campaign duration, $s$ be the total sales of all the markets, and $S_{i}$ be the total sales in market $i$ during the period $\left[t_{0}, T\right]$, where $t_{0}$ is the starting time and $T$ is the measuring time $\left(T=t_{0}+\tau, \tau \geq \theta\right)$. In a particular market $i$, the rate of sales, advertising budget, initial value of sales rate, sales decay constant, saturation level, and response constant can be symbolically represented by $s_{i}, c_{i}, \mu_{i}, \lambda_{i}, M_{i}, r_{i}$, respectively. It is obvious that $S, s, C, c, \theta, \tau, \mu, \lambda, M, r \geq 0$. Two budget decision models for multimarkets with multiconstraints are given as 


$$
\begin{aligned}
& \begin{cases}\max & S=\sum S_{i} \\
\text { s.t. } & C^{*}=\sum c_{i},\end{cases} \\
& \text { model I: }\left\{\begin{array}{l}
\frac{s_{i}(T)}{M_{i}} \geq z_{i} \quad i \in A,
\end{array}\right. \\
& p_{j} S_{j}-c_{j} \geq l_{j} \quad j \in B, \\
& C^{*}, C, \theta, \tau, c_{i}, \mu_{i}, \lambda_{i}, M_{i} \quad r_{i} \geq 0, \\
& \text { model II : } \begin{cases}\min & C=\sum c \\
\text { s.t. } & S^{*}=\sum \\
\frac{s_{i}(T)}{M_{i}} \geq z_{i} & i \in A, \\
p_{j} S_{j}-c_{j} \geq l_{j} & j \in B, \\
S^{*}, C, \theta, \tau, c_{i}, \mu_{i}, \lambda_{i}, M_{i} & r_{i} \geq 0 .\end{cases}
\end{aligned}
$$

In model I, $C^{*}$ is the total fixed budget, and in model II, $S^{*}$ is the targeted total sales volume. $A$ and $B$ represent the sets of type A and type B markets, respectively, where $|A|+$ $|B| \leq n$ indicates that the total number of markets $A$ and markets $B$ is less than or equal to the number for all markets.

The two models address different allocation optimization problems. Model I addresses the decision problem of how to maximize sales volume given a fixed total budget $C$ for multiple type A and type B markets. In contrast, model II deals with the decision problem of how to minimize advertising expenditure across two types of markets with different advertising objectives when the sales goal is given. Managers should select a proper model according to their various goals of advertising budget allocation.

\section{Theorem and Corollary for Two Types of Markets}

As there are different objectives in two types of markets, we consider some characteristics derived from conditions (9) and (10) by using Vidale-Wolfe models in this section of the two types of markets, respectively.

3.1. Characteristics of Type A Markets. Type A markets indicate new markets a company intends to break into, so it is obvious that the rate of sales at starting time $t_{0}$ is 0 , that is,

$$
\mu=s\left(t_{0}\right)=0 .
$$

According to the Vidale-Wolfe model, we can derive the rate of sales at $T=t_{0}+\tau$ from equation (7) as

$$
s(T)=\frac{M r c}{r c+\lambda M \theta}\left(e^{\lambda \theta}-e^{-(r c / M)}\right) e^{-\lambda \tau} .
$$

Then, the market-share goal (9) for type A markets can be expressed as

$$
\frac{s(T)}{M}=\frac{r c}{r c+\lambda M \theta}\left(e^{\lambda \theta}-e^{-(r c / M)}\right) e^{-\lambda \tau} \geq z .
$$

For convenience, we can rewrite equation (14) into another form as

$$
F(c)=\frac{s(T)}{M}-z=\frac{r c}{r c+\lambda M \theta}\left(e^{\lambda \theta}-e^{-(r c / M)}\right) e^{-\lambda \tau}-z \geq 0 .
$$

The function $F(c)$ indicates the difference between actual market share and expected market share after an advertising campaign. Advertising achieves the expectation only when $F(c)$ is positive. Next, we discuss some characters of the differential function.

Theorem 1. $F(c)$ is a monotonously increasing function on the domain of $[0,+\infty)$.

Proof. To prove the proposition is equivalent to showing $(\partial F(c) / \partial c) \geq 0$ on the domain of $[0,+\infty)$,

$$
\begin{aligned}
\frac{\partial F(c)}{\partial c}= & \frac{r \lambda M \theta}{(r c+\lambda M \theta)^{2}}\left(e^{\lambda \theta}-e^{-(r c / M)}\right) e^{-\lambda \tau} \\
& +\frac{r^{2} c}{M(r c+\lambda M \theta)} e^{-(r c / M)} e^{-\lambda \tau} .
\end{aligned}
$$

Apparently, all the parameters are greater than 0 , and it is easy to obtain inequalities as

$$
e^{\lambda \theta} \geq 1, \quad 0<e^{-(r c / M)} \leq 1,0<e^{-\lambda \tau} \leq 1 .
$$

Thus,

$$
\begin{aligned}
\frac{r \lambda M \theta}{(r c+\lambda M \theta)^{2}}\left(e^{\lambda \theta}-e^{-(r c / M)}\right) e^{-\lambda \tau} \geq & , \\
\frac{r^{2} c}{M(r c+\lambda M \theta)} e^{-(r c / M)} e^{-\lambda \tau} & \geq 0, \\
\frac{\partial F(c)}{\partial c}= & \frac{r \lambda M \theta}{(r c+\lambda M \theta)^{2}}\left(e^{\lambda \theta}-e^{-(r c / M)}\right) e^{-\lambda \tau} \\
& +\frac{r^{2} c}{M(r c+\lambda M \theta)} e^{-(r c / M)} e^{-\lambda \tau} \geq 0 .
\end{aligned}
$$

Thus, $F(c)$ is proved to be a monotonously increasing function.

Theorem 1 illustrates that the difference between actual market share and expected market share is increasing with the increase of advertising expenditure. As the expected market share $z$ is fixed, it is easy to know that advertising can bring the increase of market share.

In particular, if and only if $r=0$, we have

$$
\frac{\partial F(c)}{\partial c}=0 .
$$

That is, if the response constant is $0, F(c)$ is a constant, and then 


$$
s(t)=0, \quad t_{0} \leq t \leq T .
$$

Apparently, sales remain zero if the rate of sales at $t_{0}$ is zero and advertising has no effect on rate of sales. Thus, we must assume the response constant is not zero. This is a rational assumption. Consequently, for every market with response constant satisfying $r>0$, we have

$$
\frac{\partial F(c)}{\partial c}>0 \text {. }
$$

A corollary can be derived from Theorem 1:

Corollary 1. $F(c)$ is a strictly increasing function on the domain of $[0,+\infty)$ if $r>0$.

This corollary illustrates that market share shall increase with the increase in advertising expenditure for a rational market where $r>0$.

However, if the company gives an excessive expected market share, the goal may not be achieved at time $T$ no matter how much advertising expenditure is. That is, for some values of $z \in(0,1]$, the inequality $F(c) \geq 0$ may not have a solution. It is because $\left(s\left(t_{0}+\theta\right) / M\right) \leq 1$ at $t=t_{0}+\theta$, while $(s(t) / M)$ is decreasing during the time interval $\left[t_{0}+\theta, T\right]$. Subsequently, market share decreases after the advertising campaign ends. Thus, we have $(s(T) / M)<1$ at time $T$. If the value of $z$ is extremely large, say close to 1 , we cannot reach $(s(T) / M) \geq z$ at time $T$ no matter how much we invest in advertising.

According to Corollary 1, $F(c)$ reaches the maximal value when $c \longrightarrow+\infty$ :

$$
\lim _{c \longrightarrow+\infty} F(c)=\lim _{c \longrightarrow+\infty}\left[\frac{r c}{r c+\lambda M \theta}\left(e^{\lambda \theta}-e^{-(r c / M)}\right) e^{-\lambda \tau}-z\right]=e^{-\lambda(\tau-\theta)} .
$$

So, the maximal value of $F(c)$ is $e^{-\lambda(\tau-\theta)}$, and we must assume $z \leq e^{-\lambda(\tau-\theta)}$. Then, we can obtain Theorem 2 as follows.
Theorem 2. Market share can reach the expected value $z$ at time $T$ through investing in advertising if $0<z \leq e^{-\lambda(\tau-\theta)}$; however, market share cannot reach the expected value $z$ if $e^{-\lambda(\tau-\theta)}<z \leq 1$ regardless of how large advertising spending is.

When $e^{-\lambda(\tau-\theta)}<z \leq 1$, the expected market share value is too large to be achieved. Thus, the targeted share needs to be adjusted downward so that $0<z \leq e^{-\lambda(\tau-\theta)}$; when $0<z \leq e^{-\lambda(\tau-\theta)}$, we have

$$
\begin{array}{r}
F(c=0)=-z<0, \\
F(c \longrightarrow+\infty)=e^{-\lambda(\tau-\theta)} \geq 0 .
\end{array}
$$

Theorem 2 illustrates that the company must raise a rational expected market share in order to imply an advertising activity and achieve the share target.

In view of the continuity and strict monotonicity of $F(c)$, we know equation $F(c)=0$ has unique solution $c_{z}$. Therefore, we have the following.

Theorem 3. $F(c) \geq 0$ if and only if $c \geq c_{z}$.

It is easy to find the numerical solution $c_{z}$ to $F(c)=0$ using the bisection method or Newton iteration method. According to equation (15), we can obtain the following.

Corollary 2. $(s(T) / M) \geq z$ if and only if $c \geq c_{z}$.

The solution $c_{z}$ in Theorem 3 and Corollary 2 is the very value that when the advertising expenditure is $c_{z}$, the market share is just reaching the expected value, so the company can calculate the very value of expenditure to achieve the market share goal.

3.2. Characteristics of Type B Markets. A type B market is a mature market with the initial rate of sales $s\left(t_{0}\right)=\mu$, and $\mu>0$ at $t_{0}$. Solving equation (8), we can get the total sales $S$ of $\left[t_{0}, T\right]$ :

$$
\begin{aligned}
S= & \frac{M \theta(\mu \lambda M \theta+r c(\mu-M))\left(1-e^{-(r c+\lambda M \theta / M)}\right)}{(r c+\lambda M \theta)^{2}}+\frac{M r c \theta}{r c+\lambda M \theta} \\
& +\frac{r c M\left(1-e^{\lambda(\theta-\tau)}\right)+(r c \mu+\mu \lambda M \theta-r c M)\left(e^{-(r c+\lambda M \theta / M)}-e^{-(r c+\lambda M \tau / M)}\right)}{\lambda(r c+\lambda M \theta)}
\end{aligned}
$$

Hence, there is

$$
\begin{aligned}
G(c)= & p S-c=p\left[\frac{M \theta(\mu \lambda M \theta+r c(\mu-M))\left(1-e^{-(r c+\lambda M \theta / M)}\right)}{(r c+\lambda M \theta)^{2}}+\frac{M r c \theta}{r c+\lambda M \theta}\right. \\
& \left.+\frac{r c M\left(1-e^{\lambda(\theta-\tau)}\right)+(r c \mu+\mu \lambda M \theta-r c M)\left(e^{-(r c+\lambda M \theta / M)}-e^{-(r c+\lambda M \tau / M)}\right)}{\lambda(r c+\lambda M \theta)}\right]-c .
\end{aligned}
$$


The profit goal of type B markets can be rewritten as

$$
G(c) \geq l .
$$

Similar as the discussion about the expected market share, if the company proposes an excessive targeted profit, the goal may not be achieved no matter how much advertising expenditure is. To ensure that equation (25) has a solution, we need to impose a constraint on the value of targeted profit $l$ such that it is not greater than the maximal value of $G(c)$. Some properties of $G(c)$ are discussed next.

Theorem 4. For type B markets, sales volume increases monotonously, while the marginal utility of advertising decreases. That is, with respect to the advertising expenditure, $(\partial / \partial c) S(c) \geq 0$ and $\left(\partial^{2} / \partial c^{2}\right) S(c) \leq 0$.

Proof. From equation (8), we obtain $S(c)=\int_{t_{0}}^{t_{0}+\theta} s_{1}(t) \mathrm{d} t+\int_{t_{0}+\theta}^{t_{0}+\tau} s_{2}(t) \mathrm{d} t$, as well as $s_{1} \geq 0, s_{2} \geq 0$, and $t_{0}+\tau>t_{0}+\theta>t_{0}$. By properties of implicit functions of partial derivative and integration, there are

$$
\begin{aligned}
\frac{\partial}{\partial c} S(c) & =\frac{\partial \int_{t_{0}}^{t_{0}+\theta} s_{1}(t) \mathrm{d} t}{\partial c}+\frac{\partial \int_{t_{0}+\theta}^{t_{0}+\tau} s_{2}(t) \mathrm{d} t}{\partial c} \\
& =\int_{t_{0}}^{t_{0}+\theta} \frac{\partial s_{1}(t)}{\partial c} \mathrm{~d} t+\int_{t_{0}+\theta}^{t_{0}+\tau} \frac{\partial s_{2}(t)}{\partial c} \mathrm{~d} t, \\
\frac{\partial^{2}}{\partial c^{2}} S(c) & =\frac{\partial^{2} \int_{t_{0}}^{t_{0}+\theta} s_{1}(t) \mathrm{d} t}{\partial c^{2}}+\frac{\partial^{2} \int_{t_{0}+\theta}^{t_{0}+\tau} s_{2}(t) \mathrm{d} t}{\partial c^{2}} \\
& =\int_{t_{0}}^{t_{0}+\theta} \frac{\partial^{2} s_{1}(t)}{\partial c^{2}} \mathrm{~d} t+\int_{t_{0}+\theta}^{t_{0}+\tau} \frac{\partial^{2} s_{2}(t)}{\partial c^{2}} \mathrm{~d} t .
\end{aligned}
$$

We can prove equations (27) and (28) by demonstrating $\left(\partial s_{1}(t) / \partial c\right) \geq 0, \quad\left(\partial^{2} s_{1}(t) / \partial c^{2}\right) \leq 0, \quad\left(\partial s_{2}(t) / \partial c\right) \geq 0, \quad$ and $\left(\partial^{2} s_{2}(t) / \partial c^{2}\right) \leq 0$, that is, $s_{1}(t)$ and $s_{2}(t)$ are monotonously increasing with respect to $c$, while the rate of increase is decreasing. It is apparent in practice that more advertising spending leads to faster rate of sales even though marginal utilities decrease. Precise derivation of these differential inequalities is given as follows:

$$
\begin{aligned}
\frac{\partial s_{1}(t)}{\partial c}= & \frac{\lambda M^{2} \theta r}{(r c+\lambda M \theta)^{2}}\left(1-e^{-(r c+\lambda M \theta / M \theta)\left(t-t_{0}\right)}\right) \\
& -\left(\mu-\frac{M r c}{r c+\lambda M \theta}\right) \frac{r}{M \theta}\left(t-t_{0}\right) e^{-(r c+\lambda M \theta / M \theta)\left(t-t_{0}\right)},
\end{aligned}
$$

where $\left(\partial s_{1}(t) / \partial c\right)$ is descending with respect to $\mu$, and the maximal value of $\mu$ is $M$. Substitute $\mu=M$ into equation (29), and we obtain

$$
\frac{\partial s_{1}(t)}{\partial c} \geq \frac{\lambda M^{2} \theta r}{(r c+\lambda M \theta)^{2}}\left[1-e^{-(r c+\lambda M \theta / M \theta)\left(t-t_{0}\right)}\right.
$$

$$
\left.-\frac{r c+\lambda M \theta}{M \theta}\left(t-t_{0}\right) e^{-(r c+\lambda M \theta / M \theta)\left(t-t_{0}\right)}\right] .
$$

We can prove $1-e^{-(r c+\lambda M \theta / M \theta)\left(t-t_{0}\right)} \quad-(r c+\lambda M \theta$ $/ M \theta)\left(t-t_{0}\right) e^{-(r c+\lambda M \theta / M \theta)\left(t-t_{0}\right)} \geq 0$.

Let $x=(r c+\lambda M \theta / M \theta)\left(t-t_{0}\right)$ and $a(x)=1-e^{-x}$ $-x e^{-x}$; then, $(\mathrm{d} a(x) / \mathrm{d} x)=x e^{-x} \geq 0, a(0)=0$; hence, $a(x)$ is monotonously increasing and $a(x) \geq 0$.

Therefore, $\left(\partial s_{1}(t) / \partial c\right) \geq 0$.

Given that, we have

$$
\begin{aligned}
\frac{\partial^{2} s_{1}(t)}{\partial c^{2}}= & -\frac{2 \lambda M^{2} \theta r^{2}}{(r c+\lambda M \theta)^{3}}\left(1-e^{-(r c+\lambda M \theta / M \theta)\left(t-t_{0}\right)}\right) \\
& +\frac{2 \lambda M^{2} \theta r}{(r c+\lambda M \theta)^{2}} \frac{r}{M \theta}\left(t-t_{0}\right) e^{-(r c+\lambda M \theta / M \theta)\left(t-t_{0}\right)} \\
& +\left(\mu-\frac{M r c}{r c+\lambda M \theta}\right)\left(\frac{r}{M \theta}\right)^{2}\left(t-t_{0}\right)^{2} e^{-(r c+\lambda M \theta / M \theta)\left(t-t_{0}\right)}
\end{aligned}
$$

It is easy to find that $\left(\partial^{2} s_{1}(t) / \partial c^{2}\right) \leq 0$ is increasing with respect to $\mu$. Substituting $\mu=M$ into equation (31), we get

$$
\begin{aligned}
\frac{\partial^{2} s_{1}(t)}{\partial c^{2}} \leq & \frac{\lambda M^{2} \theta r^{2}}{(r c+\lambda M \theta)^{3}}\left[2\left(e^{-(r c+\lambda M \theta / M \theta)\left(t-t_{0}\right)}-1\right)+2 \frac{r c+\lambda M \theta}{M \theta}\left(t-t_{0}\right) e^{-(r c+\lambda M \theta / M \theta)\left(t-t_{0}\right)}\right. \\
& \left.+\left(\frac{r c+\lambda M \theta}{M \theta}\right)^{2}\left(t-t_{0}\right)^{2} e^{-(r c+\lambda M \theta / M \theta)\left(t-t_{0}\right)}\right] .
\end{aligned}
$$

Using a method similar to the previous one, we let $b(y)=2\left(e^{-y}-1\right)+2 y e^{-y}+y^{2} e^{-y}$. Then, $(\mathrm{d} b(x) / \mathrm{d} x)=$ $-x^{2} e^{-x} \leq 0$ and $b(0)=0$. Hence, $\quad b(x) \leq 0$, and $\left(\partial^{2} s_{1}(t) / \partial c^{2}\right) \leq 0$ is proved.

$\left(\partial s_{2}(t) / \partial c\right) \geq 0$ and $\left(\partial^{2} s_{2}(t) / \partial c^{2}\right) \leq 0$ can be proved in a similar manner.

In terms of Theorem $4,(\partial / \partial c) S(c)$ assumes the minimal value when $c \longrightarrow+\infty$ and the maximal value when $c \longrightarrow 0$. Working through some calculations, we obtain

$$
\begin{aligned}
& \lim _{c \longrightarrow+\infty} \frac{\partial}{\partial c} S(c)=0, \\
& \lim _{c \longrightarrow 0} \frac{\partial}{\partial c} S(c)=r\left(\frac{\mu}{\lambda^{2} \theta M e^{\lambda \theta}}+\frac{M+\mu \lambda \theta-M e^{\lambda \theta}}{\lambda^{2} \theta M e^{\lambda \tau}}+\frac{\lambda \theta M-\mu}{\lambda^{2} \theta M}\right) .
\end{aligned}
$$

Theorem 5. There exists $\mu_{a}$ such that when $\mu<\mu_{a}, G(c)$ is increasing at first and then decreasing on the domain of 
definition $[0,+\infty)$; when $\mu_{a} \leq \mu \leq M, G(c)$ is a monotonously decreasing function on $[0,+\infty)$.

Proof. By Theorem 4, we know that $(\partial / \partial c) S(c)$ is monotonously decreasing, and the range of values is

$$
\left[0, r\left(\frac{\mu}{\lambda^{2} \theta M e^{\lambda \theta}}+\frac{M+\mu \lambda \theta-M e^{\lambda \theta}}{\lambda^{2} \theta M e^{\lambda \tau}}+\frac{\lambda \theta M-\mu}{\lambda^{2} \theta M}\right)\right] .
$$

Hence, the range for $(\partial G(c) / \partial c)=p(\partial S(c) / \partial c)-1$ is

$$
\left[-1, \operatorname{pr}\left(\frac{\mu}{\lambda^{2} \theta M e^{\lambda \theta}}+\frac{M+\mu \lambda \theta-M e^{\lambda \theta}}{\lambda^{2} \theta M e^{\lambda \tau}}+\frac{\lambda \theta M-\mu}{\lambda^{2} \theta M}\right)-1\right] .
$$

$$
p \cdot r\left(\frac{\mu}{\lambda^{2} \theta M e^{\lambda \theta}}+\frac{M+\mu \lambda \theta-M e^{\lambda \theta}}{\lambda^{2} \theta M e^{\lambda \tau}}+\frac{\lambda \theta M-\mu}{\lambda^{2} \theta M}\right)-1<0,
$$

then,

$$
\lim _{c \longrightarrow 0} \frac{\partial}{\partial c} S(c)=r\left(\frac{\mu}{\lambda^{2} \theta M e^{\lambda \theta}}+\frac{M+\mu \lambda \theta-M e^{\lambda \theta}}{\lambda^{2} \theta M e^{\lambda \tau}}+\frac{\lambda \theta M-\mu}{\lambda^{2} \theta M}\right)<\frac{1}{p} .
$$

Thus, $-1 \leq(\partial G(c) / \partial c)<0$, and $G(c)$ is a monotonously decreasing function on $[0,+\infty)$.

If

$$
p \cdot r\left(\frac{\mu}{\lambda^{2} \theta M e^{\lambda \theta}}+\frac{M+\mu \lambda \theta-M e^{\lambda \theta}}{\lambda^{2} \theta M e^{\lambda \tau}}+\frac{\lambda \theta M-\mu}{\lambda^{2} \theta M}\right)-1>0,
$$

then,

$$
\lim _{c \longrightarrow 0} \frac{\partial}{\partial c} S(c)=r\left(\frac{\mu}{\lambda^{2} \theta M e^{\lambda \theta}}+\frac{M+\mu \lambda \theta-M e^{\lambda \theta}}{\lambda^{2} \theta M e^{\lambda \tau}}+\frac{\lambda \theta M-\mu}{\lambda^{2} \theta M}\right)>\frac{1}{p} .
$$

Based on the monotonously decreasing property of $(\partial / \partial c) S(c)$, there exists $c_{m}>0$ such that $(\partial / \partial c) S(c)=(1 / p)$ when $c=c_{m}, \quad(\partial / \partial c) S(c)>(1 / p)$ when $0 \leq c<c_{m}$, and $(\partial / \partial c) S(c)<(1 / p)$ when $c_{m}<c$. In other words, $(\partial G(c) / \partial c)=0$ when $c=c_{m},(\partial G(c) / \partial c)>0$ when $0 \leq c<c_{m}$, and $-1 \leq(\partial G(c) / \partial c)<0$ when $c_{m}<c$.

Note that, in the inequality

$$
r\left(\frac{\mu}{\lambda^{2} \theta M e^{\lambda \theta}}+\frac{M+\mu \lambda \theta-M e^{\lambda \theta}}{\lambda^{2} \theta M e^{\lambda \tau}}+\frac{\lambda \theta M-\mu}{\lambda^{2} \theta M}\right)>\frac{1}{p} .
$$

$\lambda, r, M, \theta, \tau$, and $p$ are all fixed for a specific market; thus, equation (40) is an inequality with respect to $\mu$ only. Simplifying it, we obtain

$$
\left(e^{\lambda \tau}+\lambda \theta e^{\lambda \theta}-e^{\lambda \theta} e^{\lambda \tau}\right) \mu+\left(e^{\lambda \theta}+\lambda \theta e^{\lambda \theta} e^{\lambda \tau}-e^{\lambda \theta} e^{\lambda \theta}\right) M>\frac{\lambda^{2} \theta M e^{\lambda \theta} e^{\lambda \tau}}{p r} .
$$

The coefficient of $\mu$ in the linear inequality above may take either positive or negative values. Given $1-e^{\lambda \theta} \leq 0$ and $e^{\lambda \theta} \leq e^{\lambda \tau}$, we can convert the coefficient of $\mu$ as follows:

$$
\begin{aligned}
e^{\lambda \tau}+\lambda \theta e^{\lambda \theta}-e^{\lambda \theta} e^{\lambda \tau}= & \lambda \theta e^{\lambda \theta}+\left(1-e^{\lambda \theta}\right) e^{\lambda \tau} \leq \lambda \theta e^{\lambda \theta} \\
& +\left(1-e^{\lambda \theta}\right) e^{\lambda \theta}=e^{\lambda \theta}\left(1+\lambda \theta-e^{\lambda \theta}\right) .
\end{aligned}
$$

Let $h(x)=1+x-e^{x}, \quad x \geq 0 ; \quad$ then, $\quad(\mathrm{d} / \mathrm{dx}) h(x)=$ $1-e^{x} \leq 0$, and thus, $h(x)$ is a monotonously decreasing function. Its maximal value at $x=0$ is $h(0)=0$; hence, $h(x) \leq 0$. Taking $x=\lambda \theta$, we obtain $1+\lambda \theta-e^{\lambda \theta} \leq 0$; then, $e^{\lambda \tau}+\lambda \theta e^{\lambda \theta}-e^{\lambda \theta} e^{\lambda \tau} \leq 0 . \quad$ Apparently, $\quad e^{\lambda \tau}+\lambda \theta e^{\lambda \theta}-$ $e^{\lambda \theta} e^{\lambda \tau}=0$ if and only if $\theta=\tau=0$. Because this situation is meaningless, we have $e^{\lambda \tau}+\lambda \theta e^{\lambda \theta}-e^{\lambda \theta} e^{\lambda \tau}<0$; hence, the coefficient of $\mu$ is negative.

Solving the inequality, we obtain

$$
\mu<\frac{\lambda^{2} \theta e^{\lambda \theta} e^{\lambda \tau}-\operatorname{pr}\left(e^{\lambda \theta}+\lambda \theta e^{\lambda \theta} e^{\lambda \tau}-e^{\lambda \theta} e^{\lambda \theta}\right)}{\operatorname{pr}\left(e^{\lambda \tau}+\lambda \theta e^{\lambda \theta}-e^{\lambda \theta} e^{\lambda \tau}\right)} M .
$$

Let $\mu_{a}=\left(\lambda^{2} \theta e^{\lambda \theta} e^{\lambda \tau}-\operatorname{pr}\left(e^{\lambda \theta}+\lambda \theta e^{\lambda \theta} e^{\lambda \tau}-e^{\lambda \theta} e^{\lambda \theta}\right) / \operatorname{pr}\left(e^{\lambda \tau}\right.\right.$ $\left.\left.+\lambda \theta e^{\lambda \theta}-e^{\lambda \theta} e^{\lambda \tau}\right)\right) M$; then, when $\mu<\mu_{a}, G(c)$ is a function that first increases and then decreases on the domain of definition $[0,+\infty)$; when $\mu_{a} \leq \mu \leq M, G(c)$ is monotonously decreasing on the domain of $[0,+\infty)$.

The multimarkets we are dealing with are heterogeneous. However, because these are rational markets, there would not be such extreme cases as $\lambda \longrightarrow 0, \lambda \longrightarrow 1, r \longrightarrow 0, r \longrightarrow 1$, and so on. The decision makers are also rational persons; thus, there would not be any $\theta$ or $\tau$ value that is extremely large or small. From the expression of $\mu_{a}$, we know that $\mu_{a}$ is monotonously increasing with regard to $p$. When $p$ is large, we have $\mu_{a}>M$. It can be verified that, with the assumption of rational markets and rational decision makers (i.e., no extreme cases), $\mu_{a}>M$ when $p>(1 / r)$; that is to say, $\mu<M<\mu_{a}$. When $p$ is small, we may observe $\mu_{a}<M$. However, the rate of sales for the fast-moving consumer product is relatively smaller because of the availability of a great variety of alternatives. Therefore, with the rational market assumption, $\mu<\mu_{a}<M$ always holds. That is, $G(c)$ is a function that first increases and then decreases on the domain of $[0,+\infty)$.

If irrational situations occur in a market or if the initial rate of sales $\mu$ of the market is so large that $\mu_{a} \leq \mu \leq M$, then based on the conclusion of Theorem 5 , the company would be better off not to invest in advertising in order to maximize profit. This is because $G(c)$ on the domain of $[0,+\infty)$ is monotonously decreasing, meaning that profit will decrease regardless of advertising efforts. Therefore, we need to assess market parameters $\lambda, r, \mu$, and $M$ to determine whether irrational markets exist before trying to find the solution to the model. For any irrational market, we allocate no advertising budget, namely, let $c=0$ in such markets. Then, we can take out those markets and solve the model for the rest type B markets where $\mu<\mu_{a}$.

From the analysis above, we may conclude that all type $\mathrm{B}$ markets discussed here satisfy $\mu<\mu_{a}$. That is, for each type B market, $G(c)$ is a function first increasing and then decreasing on the domain of definition $[0,+\infty)$. Theorems 4 and 5 indicate that, with the increase of advertising expenditure in type B markets, the sales volume $S$ at any time $t$ is increased, while the growth is slow, and in other words, the sales volume is monotone increasing while marginal decreasing with 
respect to advertising expenditure, and furthermore, the profit $G$ would experience an increase first and then a decrease with respect to advertising expenditure. Therefore, according to the properties of functions $S(c)$ and $G(c)$, there must exist a unique critical value of advertising expenditure, yielding that the company receives the maximal market profit. The uniqueness of the advertising expenditure can be verified as shown in Corollary 3.

Corollary 3. $(\partial G(c) / \partial c)=0$ has a unique solution $c_{m}$ on the domain of definition $[0,+\infty)$.

Apparently, $\quad \lim _{c \longrightarrow 0} S(c)=(\mu / \lambda)\left(1-e^{-\lambda \tau}\right)$; hence, $G(0)=(p \mu / \lambda)\left(1-e^{-\lambda \tau}\right)>0$. Because all parameters are greater than zero, we have $G(0)>0$. Thus, the profit that the company can achieve during the time interval $\left[t_{0}, T\right]$ would be $(p \mu / \lambda)\left(1-e^{-\lambda \tau}\right)$ even though no advertising campaign is conducted.

Therefore, $G(c)$ reaches its maximum when $(\partial G(c) / \partial c)=0$. Given the uniqueness of the solution, its solution $c_{m}$ can be found using Newton iteration method. Consequently, $G\left(c_{m}\right)$ is the maximal profit that can be achieved through advertising during the time interval $\left[t_{0}, T\right]$. Thus, when advertising expenditure reaches $c_{m}$, further increase in advertising spending may result in more sales, but the profit will gradually decline. Therefore, $c_{m}$ is the rational threshold for advertising expenditure.

Theorem 6. Ifl $\leq(p \mu / \lambda)\left(1-e^{-\lambda \tau}\right)$, profit of a type B market can reach the expected value $l$ without advertising; if $l>G\left(c_{m}\right)$, targeted profit $l$ cannot be achieved no matter how much is invested in advertising; and if $(p \mu / \lambda)\left(1-e^{-\lambda \tau}\right)<l \leq G\left(c_{m}\right)$, targeted profit l can be reached by means of advertising campaigns.

Theorem 6 provides an advice for managers that a rational profit target is essential when promoting an advertising activity: an excessive high profit target or an excessive low profit target may both result in invalidity and waste of advertising expenditure, and only a rational profit target by considering parameters of the market in advance can lead to a successful advertising activity. The solution to $G(c) \geq l$ is $c \geq 0$ if $l \leq(p \mu / \lambda)\left(1-e^{-\lambda \tau}\right) . G(c) \geq l$ has no solution if $l>G\left(c_{m}\right)$. The unique solution of $G(c) \geq l$ is $c_{m}$ if $l=G\left(c_{m}\right)$. And in the case of $(p \mu / \lambda)\left(1-e^{-\lambda \tau}\right)<l \leq G\left(c_{m}\right)$, we must first find the solution of $G(c)=l$. As $G(c)$ is a function first increasing and then decreasing, $G(c)=l$ has two solutions: $c_{l 1}$ and $c_{l 2}$ with $c_{l 1}<c_{m}<c_{l 2}$. Using Newton iteration method on $\left[0, c_{m}\right]$ and $\left[c_{m},+\infty\right)$, respectively, we can find $c_{l 1}$ and $c_{l 2}$ for $G(c)=l$. Hence, the solution to $G(c) \geq l$ is $c_{l 1} \leq c<c_{l 2}$.

If it just so happens that $l>G\left(c_{m}\right)$ in a type B market, then it is necessary to lower the expected profit value such that $l \leq G\left(c_{m}\right)$.

\section{Solutions to the Two Models and Results of a Numerical Example}

4.1. Solutions to Models. The models have solutions if and only if $0<z_{i}<e^{-\lambda_{i}(\tau-\theta)}$ and $l_{j} \leq G\left(c_{m_{j}}\right)$. Thus, it is necessary to first assess whether the values of $z_{i}$ and $l_{j}$ are in reasonable ranges. If not, managers need to adjust them before finding the solutions to the models.

According to Corollary 2, the constraints of $\left(s_{i}(T) / M_{i}\right) \geq z_{i}$ in the type A markets can be rewritten as $c_{i} \geq c_{z_{i}}$, where $c_{z_{i}}$ are numerical solutions to $\left(s_{i}(T) / M_{i}\right)=z_{i}$, and according to Theorem 6, when $\left(p_{j} \mu_{j} / \lambda_{j}\right)(1-$ $\left.e^{-\lambda_{j} \tau}\right)<l_{j} \leq G\left(c_{m_{j}}\right)$, the constraints $p_{j} S_{j}-c_{j} \geq l_{j}$ in the type B markets are equivalent to $c_{l_{1} 1} \leq c_{j} \leq c_{l_{2}, 2}$, where $c_{l_{1} 1}$ and $c_{l_{1} 2}$ are two solutions of $p_{j} S_{j}-c_{j}=l_{j}$ and satisfy $c_{l_{1} 1} \leq c_{l_{j}}$; when $l_{j} \leq\left(p_{j} \mu_{j} / \lambda_{j}\right)\left(1-e^{-\lambda_{j} \tau}\right), \quad p_{j} S_{j}-c_{j} \geq l_{j}$ are equivalent to $c_{j} \geq 0$. Symbolizing the type $\mathrm{B}$ markets where there are $\left(p_{j} \mu_{j} / \lambda_{j}\right)\left(1-e^{-\lambda_{j} \tau}\right)<l_{j} \leq G\left(c_{m_{j}}\right)$ as set $B \prime$, we can let $(B-$ $B$ I) represent the rest of the type $B$ markets where $l_{j} \leq\left(p_{j} \mu_{j} / \lambda_{j}\right)\left(1-e^{-\lambda_{j} \tau}\right)$

Then, we propose the following equivalent forms of the former two models shown in (11a) and (11b).

Model I' (seeking the maximum sales in fixed budget):

$$
\begin{cases}\max & S=\sum S_{i} \\ \text { s.t. } & C^{*}=\sum c_{i}, \\ c_{i} \geq c_{z_{i}} & i \in A, \\ c_{l_{j} 1} \leq c_{j} \leq c_{l_{j} 2} & j \in B^{\prime}, \\ C^{*}, \theta, \tau, c_{i}, \mu_{i}, \lambda_{i}, M_{i} & r_{i} \geq 0 .\end{cases}
$$

Model II' (seeking the minimum advertising expenditure in fixed sales):

$$
\begin{cases}\min & C=\sum c_{i} \\ \text { s.t } & S^{*}=\sum S_{i}, \\ c_{i} \geq c_{z_{i}} & i \in A, \\ c_{l_{j} 1} \leq c_{j} \leq c_{l_{j} 2} & j \in B^{\prime}, \\ S^{*}, \theta, \tau, c_{i}, \mu_{i}, \lambda_{i}, M_{i} & r_{i} \geq 0 .\end{cases}
$$

Therefore, solving the original models can be converted into solving the two nonlinear programming models. In model I', the objective function is nonlinear, and the constraints are linear, while in model $\mathrm{I}^{\prime}$, the objective function is linear, and the constraints are nonlinear. It would need only a simple operation to find the numerical solutions to these new models using LINGO. A numerical example for model $I^{\prime}$ is given in the following for demonstration purpose. Readers can also find the solution for model $\mathrm{II}^{\prime}$ in their own using a method similar to the method we use as follows.

4.2. Numerical Example. Company $X Y Z$ is planning for an advertising campaign across six markets labeled as A, B, C, $\mathrm{D}, \mathrm{E}$, and $\mathrm{F}$. The campaign will be conducted during the first quarter of the next year. The total budget is 7 million dollars, and the effectiveness of the advertising campaign will be assessed at the end of April (the measuring time). Markets A and $\mathrm{B}$ are new markets to the company, and the targeted market shares for the two markets at measuring time are 3\% and $2 \%$, respectively; markets $\mathrm{C}$ and $\mathrm{D}$ are mature markets, 
and the targeted profits for the first 4 months are 10 and 15 million dollars for $\mathrm{C}$ and $\mathrm{D}$, respectively, and the net profit per unit of product is 12 dollars for $C$ and 11 dollars for $\mathrm{D}$; there are no specific requirements set for markets $\mathrm{E}$ and $\mathrm{F}$. The parameters of those markets are shown in Table 2 (all the units of $M_{i}$ and $\mu_{i}$ are in millions).

The solving process is described as follows:

Step 1: building an optimal budget model based on model I as

$$
\begin{cases}\max & S=\sum S_{i} \\ \text { s.t. } & 7=\sum_{i=1}^{6} c_{i} \\ & \frac{s_{1}(4)}{6} \geq 0.03 \\ & \frac{s_{2}(4)}{8} \geq 0.02 \\ & 12 S_{3}-c_{3} \geq 10 \\ & 11 S_{4}-c_{4} \geq 15 \\ & c_{1}, c_{2}, \ldots, c_{6} \geq 0\end{cases}
$$

Step 2: transforming constraints for new markets A and B:

The maximal market shares for markets $\mathrm{A}$ and $\mathrm{B}$ are $78 \%$ and $82 \%$, respectively; thus, targeted shares $z_{1}=$ $3 \%$ and $z_{2}=2 \%$ are both in the reasonable range. We then plug the numerical value of each parameter into equation $(s(T) / M)=z$ and obtain the solution $c=0.7647$. Similarly, solving for the solution to market $\mathrm{B}$, we get $c=0.6544$. According to Corollary 2, the constraints of these two markets can be converted into $c_{1} \geq 0.7647$ and $c_{2} \geq 0.6544$, respectively.

Step 3: transforming constraints for mature markets $C$ and D:

Profits for markets C and D can reach 10.829 and 11.125 million dollars, respectively, even though no advertising campaign is conducted. Inspecting the numbers against the targeted profits, we know that there is no need to invest in advertising for market C; thus, the constraint for $\mathrm{C}$ is equivalent to $c_{3} \geq 0$. Solving $\left(\partial G\left(c_{4}\right) / \partial c_{4}\right)=0$, we can obtain that the maximal profit for market D is $G\left(c_{m_{4}}\right)=1.0421$, which is greater than the expected value. Solving $p_{4} S_{4}-c_{4}=l_{4}$, we get two solutions: $c_{41}=0.6299$ and $c_{42}=254.60$. According to Theorem 6 , the constraint for market $\mathrm{D}$ can be converted into $0.6296 \leq c_{4} \leq 254.60$.

Step 4: solving the transformed model as
TABLE 2: Parameters of each market.

\begin{tabular}{lcccc}
\hline Market & $\lambda_{i}$ & $M_{i}$ & $r_{i}$ & $\mu_{i}$ \\
\hline A $(i=1)$ & 0.25 & 6 & 0.44 & 0 \\
B $(i=2)$ & 0.20 & 8 & 0.40 & 0 \\
C $(i=3)$ & 0.15 & 9 & 0.39 & 0.03 \\
D $(i=4)$ & 0.25 & 7 & 0.38 & 0.04 \\
E $(i=5)$ & 0.25 & 10 & 0.42 & 0.01 \\
F $(i=6)$ & 0.20 & 3 & 0.45 & 0.01 \\
\hline
\end{tabular}

$$
\begin{cases}\max & S=\sum_{i=1}^{6} S_{i} \\ \text { s.t. } & 5=\sum_{i=1}^{6} c_{i}, \\ & c_{1} \geq 0.7647, \\ & c_{2} \geq 0.6544, \\ & 0.6296 \leq c_{4} \leq 254.60, \\ & c_{3}, c_{5}, c_{6} \geq 0 .\end{cases}
$$

Using LINGO v16.0, we can obtain the optimal results as $c_{1}=0.880, \quad c_{2}=1.285, \quad c_{3}=1.831, \quad c_{4}=0.998$, $c_{5}=1.414$, and $c_{6}=0.592$, with the optimal objective $S=25.14$. Thus, the advertising budgets of the 6 markets are $0.88,1.285,1.831,0.998,1.414$, and 0.592 million dollars, respectively, and in this case, the company can obtain the maximal sales which is 25.14 million.

\section{Concluding Comments}

Advertising budget allocation across multiple markets has drawn considerable attention from both scholars and practitioners in recent years. To fill a gap in the current literature, we have developed two decision models for optimal budget allocation for multimarkets with different market goals, and consequently different advertising objectives. The contributions of the present study are threefold.

First, similar to previous research, this study bases advertising allocation decisions on the consideration of heterogeneous market conditions, particularly in terms of market saturation level, sales decay constant, and consumer responses to adverting. Unlike previous research, this study incorporates market goals (strategic consideration) and advertising objectives into allocation decisions. Rather than making profit maximization, the only goal for all the markets in question, we explicitly include targeted share as a market goal besides profit maximization while examining different types of markets. Just as marketing strategies must be guided by corporate strategies and objectives, advertising allocation decisions must take into account corporate market goals as well as advertising objectives set for different markets on the basis of heterogeneous market conditions. This approach offers us a unique perspective when looking at the market 
differences and subsequently making budget allocation decision.

Secondly, considering the different market characteristics of both type A and type B markets, we developed two different models to accommodate the differences in market goals for two types of markets. Each of the models addresses a unique decision problem. As a result, the objective of advertising budget allocation is broken into two separate decision problems: maximizing sales with a fixed budget and minimizing advertising expenditure with a predetermined sales objective. In other words, with the models presented here, optimization of budget allocation no longer aims only at profit maximization or maximizing the present value of the profit. The varying objectives for budget allocation enable us to consider optimal decisions from different perspectives so that optimization need not be solely defined based on profit consideration.

Finally, although the two models address different decision problems, each model here takes consideration of different constraints determined by the types of markets with varying market goals and advertising objectives. Incorporating different constraints and varying market goals and objectives into the models, we believe the models better approximate the actual budget allocation decisions for multimarkets. By releasing the assumption of profit maximization being the goal for all markets, the decision models presented here are more realistic and consequently should have a broad application in budget allocation decisions. These models should be of significant assistance to practitioners, especially managers of transnational companies.

In short, this study might be the first attempt examining multimarket advertising allocation decisions from a multiconstraint perspective while considering different market goals for different types of markets. For future research, scholars may want to further look into other types of constraints in terms of differences in market goals or objectives. Given the multitude of market goals and corporate objectives, there is a need for other forms of multiconstraint models. Moreover, future research may investigate multimarket budget allocation problems in terms of different advertising modes and consumer response patterns that have been shown to influence sales and characters of markets. Research opportunity abounds in regard to multimarket advertising budget allocation.

\section{Data Availability}

All data included in this study are available upon request by contact with the corresponding author.

\section{Conflicts of Interest}

The authors declare no conflicts of interest.

\section{Acknowledgments}

This study was supported by the Key Technology Research and Development Program of Shandong (no. 2016CYJS1A01-3), the National Natural Science Foundation of China (no.
71272121), the Shandong Soft Science Project (no. 2017RZE27004), the Humanities and Social Sciences Research Project of Ministry of Education of China (no. 19YJC630059), the Natural Science Foundation of Shandong Province (no. ZR2019PG009), and the Higher Educational Social Science Program of Shandong Province (no. J18RA112).

\section{References}

[1] M. L. Vidale and H. B. Wolfe, "An operations-research study of sales response to advertising," Operations Research, vol. 5, no. 3, pp. 370-381, 1957.

[2] R. Dorfman and P. O. Steiner, "Optimal advertising and optimal quality," The American Economic Review, vol. 44, no. 5, pp. 826-836, 1954.

[3] M. Nerlove and K. J. Arrow, "Optimal advertising policy under dynamic conditions," Economica, vol. 29, no. 114, pp. 129-142, 1962.

[4] S. P. Sethi, "Deterministic and stochastic optimization of a dynamic advertising model," Optimal Control Applications and Methods, vol. 4, no. 2, pp. 179-184, 1983.

[5] S. P. Sethi, A. Prasad, and X. He, "Optimal advertising and pricing in a new-product adoption model," Journal of Optimization Theory and Applications, vol. 139, no. 2, pp. 351-360, 2008.

[6] D. G. Clarke, "Econometric measurement of the duration of advertising effect on sales," Journal of Marketing Research, vol. 13, no. 4, pp. 345-357, 1976.

[7] L. Friedman, "Game-theory models in the allocation of advertising expenditures," Operations Research, vol. 6, no. 5, pp. 699-709, 1958.

[8] J.-J. Lambin, "Measuring the profitability of advertising: an empirical study," The Journal of Industrial Economics, vol. 17, no. 2, pp. 86-103, 1969.

[9] M. W. Sasieni, "Optimal advertising expenditure," Management Science, vol. 18, no. 4, 1971.

[10] A. Mihiotis and I. Tsakiris, "A mathematical programming study of advertising allocation problem," Applied Mathematics and Computation, vol. 148, no. 2, pp. 373-379, 2004.

[11] H. I. Mesak and T. L. Means, "Modelling advertising budgeting and allocation decisions using modified multinomial logit market share models," Journal of the Operational Research Society, vol. 49, no. 12, pp. 1260-1269, 1998.

[12] J. D. C. Little, "Feature article-aggregate advertising models: the state of the art," Operations Research, vol. 27, no. 4, pp. 629-667, 1979.

[13] A. Krishnamoorthy, A. Prasad, and S. P. Sethi, "Optimal pricing and advertising in a durable-good duopoly," European Journal of Operational Research, vol. 200, no. 2, pp. 486-497, 2010.

[14] M. Fisher, S. Albers, N. Wagner, and M. Frie, "Dynamic marketing budget allocation across countries, Products, and marketing activities," Marketing Science, vol. 30, no. 4, pp. P568-P585, 2011.

[15] J. Zhang, L. Lei, S. Zhang, and L. Song, "Dynamic vs. static pricing in a supply chain with advertising," Computers \& Industrial Engineering, vol. 109, pp. 266-279, 2017.

[16] B. J. Bronnenberg and V. Mahajan, "Unobserved retailer behavior in multimarket data: joint spatial dependence in market shares and promotion variables," Marketing Science, vol. 20, no. 3, pp. 284-299, 2001.

[17] G. Tragler, "Optimal controls in spatial advertising diffusion models," Optimization, Dynamics, and Economic Analysis, pp. 288-297, 2000. 
[18] D. S. Tull, V. R. Wood, D. Duhan, T. Gillpatick, K. R. Robertson, and J. G. Helgeson, "Leveraged" decision making in advertising; the flat maximum prinicple and its implicaitons," Journal of Marketing Research, vol. 23, no. 1, pp. P25-P32, 1986.

[19] V. Kumar, R. Venkatesan, T. Bohling, and D. Beckmann, "The power of CLV: managing lifetime value at IBM," Marketing Science, vol. 27, no. 4, pp. P585-P599, 2008.

[20] W. Reinartz, J. S. Thomas, and V. Kumar, "Balancing acquisition and retention resources to maximize customer profitability," Journal of Marketing, vol. 69, no. 1, pp. 63-79, 2005.

[21] A. A. Zoltners and P. Sinha, "Sales territory design: third years of modeling and implementation," Marketing Science, vol. 24, no. 3, pp. P313-P331, 2005.

[22] V. S. Abedi, "Allocation of advertising budget between multiple channels to support sales in multiple markets," Journal of the Operational Research Society, vol. 68, no. 2, pp. 134-146, 2017.

[23] S. M. Muneeb, A. Y. Adhami, Z. Asim, and S. A. Jalil, "Bi-level decision making models for advertising allocation problem under fuzzy environment," International Journal of System Assurance Engineering and Management, vol. 10, no. 2, pp. 160-172, 2019.

[24] S. Eshghi, V. Preciado, S. Sarkar et al., "Spread, then target, and advertise in waves: optimal budget allocation across advertising channels," IEEE Transactions on Network Science and Engineering, 2019.

[25] A. Aravindakshan, K. Peters, and P. A. Naik, "Spatiotemporal allocation of advertising budgets," Journal of Marketing Research, vol. 49, no. 1, pp. 1-14, 2012.

[26] J.-P. Dubé and P. Manchanda, "Differences in dynamic brand competition across markets: an empirical analysis," Marketing Science, vol. 24, no. 1, pp. 81-95, 2005.

[27] G. Liu, J. Zhang, and W. Tang, "Strategic transfer pricing in a marketing-operations interface with quality level and advertising dependent goodwill," Omega, vol. 56, no. 10, pp. 1-15, 2015.

[28] L. Feng, J. Zhang, and W. Tang, "A joint dynamic pricing and advertising model of perishable products," Journal of the Operational Research Society, vol. 66, no. 8, pp. 1341-1351, 2015.

[29] L. Lu, Q. Gou, W. Tang, and J. Zhang, "Joint pricing and advertising strategy with reference price effect," International Journal of Production Research, vol. 54, no. 17, pp. 5250-5270, 2016.

[30] W. Aliaga, A. Bhaya, and E. Kaszkurewicz, "Dynamics of duopoly models with undecided clients under decentralized affine feedback advertising policies," Complexity, vol. 2020, Article ID 5371492, 11 pages, 2020.

[31] Z. Wu, L. Feng, and D. Chen, "Coordinating pricing and advertising decisions for supply chain under consignment contract in the dynamic setting," Complexity, vol. 2018, Article ID 7697180, 11 pages, 2018.

[32] F. Lu, G. Liu, J. Zhang, and W. Tang, "Benefits of partial myopia in a durable product supply chain considering pricing and advertising," Journal of the Operational Research Society, vol. 67, no. 10, pp. 1309-1324, 2016.

[33] F. Lu, W. Tang, G. Liu, and J. Zhang, "Cooperative advertising: a way escaping from the prisoner's dilemma in a supply chain with sticky price," Omega, vol. 86, no. 7, pp. 87-106, 2019.

[34] F. Lu, J. Zhang, and W. Tang, "Wholesale price contract versus consignment contract in a supply chain considering dynamic advertising," International Transactions in Operational Research, vol. 26, no. 5, pp. 1977-2003, 2019.

[35] J. Nie and J. Zhang, "Distribution channel selection considering advertising productiveness," IMA Journal of Management Mathematics, vol. 28, pp. 437-450, 2015.

[36] P. Zhang and X. Y. Wang, "A decision model for multi-market advertising budget allocation based on vidale-wolfe model," Chinese Journal of Management Science, vol. 21, no. 4, pp. 8-16, 2013. 